1 Universidade Estadual do Rio de Janeiro (Uerj), Instituto de Medicina Social, Observatório de Recursos Humanos em Saúde - Rio de Janeiro, Brasil.

marcia.ney.unesa@gmail.com

2 Universidade Estadual do Rio de Janeiro, Instituto de Medicina Social - Rio de Janeiro (RJ), Brasil. cpierantoni@gmail.com

3 Universidade Nova de Lisboa, Instituto de Higiene e Medicina Tropical Lisboa, Portugal. luis.lapao@ihmt.unl.pt

\section{Sistemas de avaliação profissional e contratualização da gestão na Atenção Primária à Saúde em Portugal}

\author{
Professional evaluation systems and contracting of the management \\ in Primary Health Care in Portugal
}

Márcia Silveira Ney¹, Celia Regina Pierantoni2, Luís Velez Lapão ${ }^{\mathbf{3}}$

RESUMO Apresenta-se um estudo de caso realizado em Portugal sobre sistemas de avaliação profissional para atenção primária. Os objetivos foram analisar a trajetória de implantação, a estrutura organizacional e as ferramentas de gestão utilizadas, bem como discutir sobre os processos que vêm sendo instituídos no Brasil com o Programa Nacional de Melhoria do Acesso e da Qualidade. Trata-se de estudo descritivo, com investigação bibliográfica de abordagem qualitativa. Os resultados indicam uma estrutura de coordenação, com descentralização dos serviços, que requer maior autonomia dos gestores de saúde e regulamentação do processo avaliativo, contribuindo para a obtenção de melhores resultados.

PALAVRAS-CHAVE Avaliação de desempenho profissional; Atenção Primária à Saúde; Administração de recursos humanos em saúde.

ABSTRACT It presents a case study performed in Portugal on professional evaluation systems in primary care. The objectives were to analyze the implanting trajectory, organizational structure and the management tools employed, as well as to discuss about the processes that have been instituted in Brazil along with National Program for Improving Access and Quality (PMAQ). It is about a descriptive study, with bibliographical investigation of qualitative approach. Results indicate a coordination structure with decentralization of services that requires greater autonomy of the health managers and regulation of the evaluation process, contributing for obtaining greater results.

KEYWORDS Employee performance appraisal; Primary Health Care; Health personnel management. 


\section{Introdução}

Diversas transformações vêm ocorrendo no Brasil e no mundo, nos últimos anos, visando à reestruturação dos serviços de saúde e das políticas que envolvem o desenvolvimento de recursos humanos e seus setores de trabalho. Há, finalmente, o reconhecimento de que o desempenho dos recursos humanos é um dos determinantes fundamentais do desempenho dos sistemas de saúde.

No Relatório da Organização Mundial da Saúde (OMS), de 2006, intitulado 'Trabalhando juntos pela saúde', foram levantadas questões sobre avaliação dos trabalhadores no âmbito da gestão de desempenho dos serviços de saúde. Segundo o relatório, o desempenho da força de trabalho é fundamental porque tem um impacto imediato na prestação de serviços e, em última instância, na saúde da população. Uma força de trabalho com bom desempenho é aquela com boa capacidade de respostas, eficiente e que atinge os melhores resultados na saúde em face dos recursos disponíveis e das circunstâncias encontradas. Há de se considerar que o desempenho da força de trabalho pode variar de acordo com algumas circunstâncias, e que os trabalhadores também podem apresentar desempenhos diferentes nos serviços (OMS, 2006).

Reconhece-se, também, que os sistemas de saúde e os determinantes de sua evolução são complexos. Devido às mudanças demográficas globais, por um lado, lidamos com o aumento da esperança de vida, com a redução da mortalidade infantil, com melhoria dos indicadores sociais da população, e, por outro, com o envelhecimento populacional, o aumento da prevalência de doenças crônicas, os desafios de garantir acesso aos cuidados de saúde à população, de reduzir os custos, de qualificar e melhorar o desempenho dos profissionais, de alcançar e gerir melhor os resultados, de trabalhar em equipe multiprofissional, de integrar uma rede de serviços, entre tantos outros (SOUSA, 2009).
Além disso, com o atual crescimento dos gastos com saúde, assumidamente uma preocupação mundial, essa discussão tem estimulado pesquisadores e formuladores de políticas a procurar soluções inovadoras sobre formas mais adequadas de utilização dos recursos, a fim de garantir que a evolução das necessidades de cuidados de saúde seja atendida e que os sistemas de saúde funcionem de forma mais eficiente e eficaz (KALINICHENKO, 2013).

Mesmo para países desenvolvidos que apresentam gastos públicos elevados no setor, como os europeus, isso ainda é um grande desafio. Em recente pesquisa, realizada em países integrantes da Organização para a Cooperação e o Desenvolvimento Econômico (OCDE), constatou-se que, se nada for feito para alterar essa situação, prevê-se que grande parte dos países irá gastar valores acima de $20 \%$ do PIB até 2050 (KALINICHENKO, 2013).

De certa forma, muito tem sido feito com a priorização de reformas dos sistemas de saúde voltadas para a Atenção Primária à Saúde (APS). Experiências exitosas enfocam questões relacionadas à melhoria de acesso à saúde, redistribuição de profissionais de saúde, sistemas mais equitativos, inclusivos e justos, mas, também, a mudanças fundamentais com o desempenho dos sistemas (VAN LEMBERGUE, 2008).

Alguns autores partilham desse interesse renovado pela APS, e relatam, ainda, que investimentos são necessários para apoiar a reforma e que esta diz respeito não apenas aos recursos humanos e à infraestrutura, mas, também, à formação, educação, investigação, melhoria contínua da qualidade, a sistemas de gestão eficazes, quadro regulamentar adequados e incentivos financeiros. Consequentemente, a melhoria contínua da qualidade na APS tem sido reconhecida por várias entidades internacionais como uma das prioridades (LAPÃO; DUSSAULT, 2012; PISCO, 2011, P. 2842).

Neste sentido, no cenário brasileiro, no que tange à agenda estratégica do SUS para a 
atenção primária, a prioridade é a Estratégia Saúde da Família (ESF) - que teve como antecessor o Programa de Agentes Comunitários de Saúde (Pacs), de 1991 -, implantada em 1994, sendo impulsionada e incentivada pelo governo (BRASIL, 2011A; VIANA, DAL POZ, 1998).

O Brasil apresentou, nos últimos anos, grande avanço no acesso e na oferta de serviços de saúde de atenção primária com sua implantação. Segundo dados do Departamento de Atenção Básica (DAB), do Ministério da Saúde (MS), em 2012, a ESF atingiu uma cobertura de $54 \%$ da população, com mais de 33 mil equipes, garantindo assistência para 124 milhões de brasileiros (BRASIL, 2012).

Com a consolidação e a expansão da estratégia, mudanças incrementais no sistema foram surgindo, como iniciativas de fortalecimento da articulação intersetorial e abertura de novos postos de trabalho, possibilitando a inserção de mais categorias profissionais, como a implantação das equipes de saúde bucal, em 2000, e a criação dos Núcleos de Apoio à Saúde da Família (Nasf), em 2008, mudanças no perfil e na formação profissional, revelando-se um programa de caráter um tanto inovador para a política de atenção à saúde do País.

Diante da expansão dos serviços, o Ministério da Saúde, em conjunto com as secretarias municipais, estaduais e instituições de ensino e pesquisa, desencadearam ações estratégicas com vistas a investimentos em atividades de monitoramento e avaliação, de forma sistemática, favorecendo a definição de uma política nacional de avaliação e monitoramento para APS no País (BRASIL, 2011B).

Diversas pesquisas vêm sendo realizadas desde então, com diferentes abordagens, em prol de melhoria de qualidade da APS brasileira. O impacto da ESF pode ser descrito como indutor da redução de hospitalizações por condições sensíveis à atenção primária e também tem sido responsável, com grande visibilidade, pela redução da mortalidade infantil no País. Os resultados alcançados nos últimos anos são marcantes para um país extenso, complexo, com características demográficas distintas e grande desigualdade social (MENDONÇA, 2009; VICTORA ET AL., 2011; MACINKO ET AL., 2007).

Porém, ainda persistem lacunas significativas na área de gestão do trabalho e recursos humanos quando se analisa o desenvolvimento de políticas voltadas para a qualificação do trabalho, atração e fixação de profissionais, tais como avaliação de desempenho, utilização de ferramentas de gestão e mecanismos de negociação do trabalho, que deem conta do contingente de profissionais que adentram diariamente em um mercado em ascensão.

Segundo pesquisa realizada em 2011 pela Estação de Pesquisa - Sinais de Mercado, do Núcleo de Educação em Saúde Coletiva/ Universidade Federal de Minas Gerais -, estima-se que a ESF seja responsável por algo próximo a 420 mil postos de trabalho em todo o País (NEY; RODRIGUES, 2012; GIRARDI ET AL., 2009).

Esforços têm sido empreendidos pelo governo no sentido de ajustar as estratégias prioritárias para o SUS e de conhecer a qualidade dos serviços ofertados na atenção primária à sociedade, nos mais diversos contextos existentes no País. Dessa forma, o Ministério da Saúde, através da publicação, em 2011, da Portaria GM/MS n. ${ }^{0} 1654$, avançou nessa direção, instituindo, no âmbito do SUS, o Programa Nacional de Melhoria do Acesso e da Qualidade da Atenção Básica (PMAQ-AB), que, além de ter como objetivo induzir à melhoria do acesso aos serviços, possa garantir qualidade, incluindo incentivos financeiros aos municípios participantes (BRASIL, 2011B).

Há de se destacar que o processo de avaliação que vem sendo preconizado no Brasil decorre de uma longa trajetória de experiências e iniciativas tomadas ao logo dos anos desde a criação da estratégia para regimento da política nacional. Também, modelos utilizados em diversos países têm sido utilizados para comparação, apoio e discussão, 
ampliando conceitos fundamentais para a construção de um modelo nacional (CASTRO; MACHADO, 2012).

$\mathrm{O}$ presente artigo insere-se nesse contexto, buscando como objeto ampliar os conhecimentos na área, analisar a trajetória de implantação de uma experiência inovadora relacionada aos processos de gestão direcionados à avaliação de desempenho e contratualização das equipes no âmbito da APS. Neste sentido, optou-se pela realização de um estudo de caso sobre o modelo de avaliação e contratualização implementado na atenção primária, em Portugal.

Os principais objetivos do estudo foram conhecer a trajetória de implantação, analisar o contexto geral e as condicionalidades de implantação do sistema, identificar os fatores facilitadores e limitantes na implantação do processo de gestão para a atenção primária, as ferramentas utilizadas, bem como obter subsídios de correlações com o programa brasileiro que vem sendo instituído atualmente.

Essa análise não prevê quaisquer comparações entre os países Brasil e Portugal, já que pontua dois sistemas de saúde diferentes, cada um com a sua história, sua reforma e sua conquista. Porém, examinar uma experiência mais 'antiga', torna sua análise interessante como propósito de enriquecer o debate, indicando um leque de alternativas e possíveis subsídios para a recente experiência, no cenário brasileiro, com o PMAQ-AB.

O artigo está estruturado iniciando-se com uma apresentação sobre a trajetória de implantação da atenção primária em Portugal; posteriormente, são discutidas as políticas para introdução de novas ferramentas de gestão, como o sistema de avaliação e contratualização para as Unidades de Saúde Familiar (USF), bem como os resultados alcançados até o momento. Em seguida, são apresentadas algumas considerações para o cenário brasileiro, finalizando com a conclusão.

\section{Metodologia}

Pesquisa de abordagem qualitativa, que envolve análise bibliográfica e documental através de coleta de documentos expedidos pelo governo de Portugal sobre a reforma da APS, estudos e pesquisas realizadas sobre o processo de contratualização e avaliação de desempenho nas USF.

Para contextualizar e atender aos objetivos do estudo, de analisar tanto o sistema nacional de saúde português quanto as suas condicionalidades no processo de implantação das ferramentas de gestão para atenção primária, foi realizado um levantamento de documentos expedidos pelo governo de Portugal, disponibilizados através do site do departamento da Direção Geral da Administração e do Emprego Público (DGAEP), da Direção Geral da Saúde (DGS), do Ministério da Saúde e do Alto Comissariado da Saúde. As normas, os decretos e as portarias vigentes para os Cuidados de Saúde Primários foram consultados através de acesso documental disponibilizado pelo link do Ministério da Saúde do País.

O levantamento bibliográfico também se apoiou na busca sistemática de artigos científicos mais recentes na área, utilizando as bases de busca Medline e Lilacs. Os descritores utilizados no estudo foram: 'atenção primária à saúde', 'cuidados de saúde primários', 'saúde da família', 'pagamento por resultados', 'contratualização', 'avaliação de desempenho', 'avaliação de processos (cuidados de saúde)', 'incentivos profissionais' e 'gestão de qualidade'; e descritores na língua inglesa: 'primary health care', 'primary healthcare reform', 'pay for performance', 'employee performance appraisal', 'management for result'. Também foi realizada leitura de teses e dissertações disponíveis no portal da Capes Periódicos e no repositório da Universidade Nova de Lisboa, bem como de relatórios disponibilizados pelo Observatório de Saúde Português e pelo Observatório Europeu dos Sistemas e Políticas de Saúde. 
Quanto à legislação e às normas vigentes para o sistema de avaliação profissional e a contextualização histórica da APS em Portugal, o trabalho levou em consideração a legislação expedida ao longo do período de 1990 e 2012.

Quanto à análise sobre a estruturação e a organização das unidades, foram examinados instrumentos normativos, como: resoluções, decretos de lei e despachos, a citar: a Resolução de Conselho de Ministro n. ${ }^{0} 157 / 2005$, que criou a estrutura de Missão para os Cuidados de Saúde Primários; o regulamento de implantação das USF; o Decreto de lei n. ${ }^{\circ}$ 28/2008, que criou os Agrupamentos de Centros de Saúde como uma nova estrutura de gestão.

$\mathrm{O}$ artigo faz parte de uma pesquisa de doutorado, com modalidade sanduíche, realizada em Lisboa/Portugal. A pesquisa foi submetida à apreciação do Comitê de Ética em Pesquisa (CEP) da Universidade do Estado do Rio de Janeiro (Uerj), de acordo com a Resolução n. ${ }^{\circ}$ 196/96, do Conselho Nacional de Saúde (CNS), e aprovada sob o parecer de número 153.996.

\section{O sistema de avaliação profissional e a contratualização na atenção primária em Portugal}

Nos últimos 30 anos, as políticas de saúde em Portugal - inserido em um contexto mundial em que todos os países iniciaram processos de reforma da atenção primária - produziram intensas transformações na trajetória, na configuração e na organização dos sistemas de saúde. Diversas foram as modificações e resoluções tomadas, possibilitando um progresso significativo e melhoria sistemática dos serviços reconhecidos internacionalmente (BRANCO; RAMOS, 2001).

Portugal foi um dos primeiros países europeus a adotar políticas integradas para organizar uma rede de serviços para os Cuidados de Saúde Primários, de uma forma generalizada, colocando os centros de saúde como o pilar central do sistema. Segundo Pisco (2011), para o sistema de saúde português, os centros de saúde são um patrimônio cultural, técnico e institucional, que importa não apenas preservar, mas, também, modernizar e desenvolver, pois continuam a ser o meio mais eficiente e acessível para proteger e promover a saúde da população (PISCO, 2011, P. 2842).

As primeiras iniciativas relacionadas aos cuidados primários datam de 1962. O País apresentava uma alta taxa de mortalidade infantil (77,5\%), sendo implementado nessa ocasião um programa de vacinação e o desenvolvimento de serviços destinados ao atendimento materno-infantil em todas as regiões do País, com o apoio da Igreja Católica e de uma rede de serviços de saúde, através das Santas Casas de Misericórdia. Essa iniciativa culminou com a intensa regressão da mortalidade infantil no País, representando um importante avanço nos últimos trinta anos, reconhecido internacionalmente, conforme registrado no relatório da Organização Mundial de Saúde sobre as reformas implantadas na APS (LAPÃO, 2012).

No entanto, ao longo dos anos, aspectos relacionados a fragilidades no processo de avaliação e monitoramento da expansão dos serviços de saúde se evidenciaram. O modelo de gestão e a organização da prestação de cuidados eram fragilizados. A excessiva centralização dos serviços nas sub-regiões e nas Administrações Regionais de Saúde (ARS), a crescente desproporção entre recursos humanos existentes na atenção primária e secundária e a ausência de um sistema remuneratório sem incentivo adequado e proporcional ao desempenho conduziram a uma deterioração da capacidade de prestação de cuidados e a uma crescente desmotivação dos profissionais.

Uma das primeiras iniciativas para reduzir o descontentamento dos profissionais e ainda possibilitar a expansão da atenção 
primária para a população foi a aprovação do regime das carreiras médicas, possibilitando aos médicos alargar o regime de trabalho, admitindo-se e motivando a prática do regime de dedicação exclusiva na atenção primária. Dessa forma, várias experiências para modificação da forma remuneratória do sistema foram iniciadas, resultando na Reforma dos Cuidados de Saúde Primários (PISCO, 2011).

Em 1996, uma experiência voltada para a mudança do processo remuneratório e organizacional das unidades de cuidados de saúde primários ocorreu, ainda que de forma localizada na região de Lisboa e Vale do Tejo, denominada Projeto Alfa. Essa iniciativa previa novas modalidades de organização e de gestão por meio das equipes de médicos de família, que deveriam alinhar sua prática em objetivos negociados com a Autoridade Regional de Saúde, a fim de melhorar o acesso e a qualidade dos serviços.

Em 1998, foi implantado pelo Ministério da Saúde o Regime Remuneratório Experimental (RRE) - Decreto-Lei n. ${ }^{\circ} 117 / 98$, de 5 de maio - para um grupo de médicos da carreira de clínica geral, médicos de família, com objetivo de organizar a prestação dos cuidados, através de adequado e justo reconhecimento dos diferentes níveis do desempenho dos profissionais. A remuneração dos médicos passava a ser baseada em um componente fixo e outro baseado em variáveis, como a realização de cuidados domiciliários, o alargamento do período assistencial, a realização de vigilância a grupos vulneráveis (gravidez, puerpério, crianças no primeiro ano e planejamento familiar), entre outros (PORTUGAL, 1998).

Com a necessidade de descentralizar a gestão das unidades e organizar os serviços de saúde pública nos níveis regional e local, foram criados os Centros de Responsabilidade Integrados (CRI), nos hospitais do Serviço Nacional de Saúde, e as agências de contratualização dos serviços de saúde, visando à distinção entre prestação e financiamento dos serviços de saúde.
Também foi estabelecido o regime dos Sistemas Locais de Saúde (SLS), como um conjunto de recursos articulados e organizados segundo critérios geográfico-populacionais, facilitando a participação social e a racionalização para a utilização dos recursos na saúde (BRANCO; RAMOS, 2001; PORTUGAL, 2012A).

Em 2005, foi criada uma estrutura de Missão para a Reforma dos Cuidados de Saúde Primários (MCSP), através da publicação de Resolução do Conselho de Ministros n. ${ }^{0} 157 / 2005$, de 22 de setembro, para conduzir o projeto de lançamento, coordenação e acompanhamento da estratégia de reconfiguração dos centros de saúde e implementação das USF. Tratava-se de um novo modelo de governação da saúde. Essa estrutura possibilitou a introdução de mecanismos gerenciais e de contratualização nas unidades de saúde (PORTUGAL, 2005).

Nessa perspectiva, a MCSP publicou, em 2006, as 'Linhas de ação prioritária para o desenvolvimento dos cuidados de saúde primários', onde divulgou propostas em oito áreas de atuação e apresentou qual seria a evolução da reforma. Estabeleceu como grandes objetivos para a reforma da APS a obtenção de mais e melhores cuidados de saúde para os cidadãos, aumento da acessibilidade e consequente aumento da satisfação dos utilizadores dos serviços. Pretendeu-se, também, aumentar a satisfação dos profissionais criando boas condições de trabalho, melhorando a organização e recompensando as boas práticas. Simultaneamente, melhorando a eficiência e promovendo a contenção de custos (PISCO, 2007).

Em setembro de 2006, foram implantadas as primeiras USF, através da deliberação do Despacho Normativo n. ${ }^{\circ}$ 9/2006, de 16 de fevereiro. Foi estabelecido o regimento jurídico para organização e funcionamento e o regime de incentivos atribuído aos seus elementos, possibilitando o alargamento da cobertura populacional na atenção primária no País.

A organização e a distribuição das USF respeitavam uma área territorial estabelecida, 
composta por três a oito médicos de família, enfermeiros e secretários clínicos, abrangendo uma população de 4000 até 14000 pessoas, e cada médico de família teria sob sua responsabilidade uma lista de pacientes cadastrados.

Nessa fase, um grande passo se estabeleceu com a introdução de ferramentas gerenciais importantes para o monitoramento das unidades, com um sistema de pagamento misto (capitação, salário, objetivos), incentivos financeiros e profissionais com recompensa ao mérito (baseado em atividades) e sensível à produtividade e à acessibilidade, mas, também, à qualidade, através de uma metodologia de contratualização estabelecida (PISCO, 2007).

A introdução do processo de contratualização com as USF foi um dos pontos fundamentais da reforma em Portugal. A gestão por objetivos das USF, através da negociação de metas, corresponde a um maior nível de exigência e de responsabilização das equipes, com vista à melhoria da prestação de cuidados, ganhos de eficiência e de satisfação dos usuários (PORTUGAL, 2008).

Com a estruturação e a organização das USF, estavam previstos três modelos de desenvolvimento, classificados de acordo com o grau de desempenho no sistema: modelo A, B ou C. A classificação de uma USF num dado nível era determinada pelo cumprimento de critérios de acordo com a seguinte diferenciação: grau de autonomia organizacional; a diferenciação do modelo retributivo e de incentivos dos profissionais; e o modelo de financiamento e seu respectivo estatuto jurídico.

Atualmente, existem apenas os modelos A e B em funcionamento. A contratualização com as USF dos modelos A e B faz-se através da definição de metas a atingir. As metas fixadas não são quantificadoras do número de consultas ou de outros atos. São um conjunto de indicadores de processo e de resultado, orientados para ganhos em saúde. Os indicadores relacionam-se com pacientes vulneráveis (crianças, grávidas) e de risco (diabéticos, hipertensos), com a acessibilidade dos cuidados, com a satisfação dos utentes e, ainda, com uma vertente econômica focada no custo dos medicamentos e Métodos Complementares de Diagnose e Terapia (MCDT).

A contratualização contempla uma carteira básica de serviços, comum a todas as USF e uma carteira adicional de serviços, aplicável apenas às USF do modelo B. A monitorização e a avaliação dos resultados culminam na atribuição dos incentivos institucionais previstos para esses dois modelos de desenvolvimento (PORTUGAL, 2008; PORTUGAL, 2010A).

Ao longo dos anos de existência das USF, a contratualização comportou alterações e ajustes próprios de um sistema implementado pela primeira vez. Na carteira básica de serviços, referente ao ano de 2007 , foram contratualizados vinte indicadores escolhidos a partir de uma lista de quarenta e nove: quinze comuns a todas as USF e cinco selecionados pelas USF. Em 2008, a Portaria n. ${ }^{\circ} 301 / 2008$, de 18 de abril, regulamentou o processo de contratualização e de atribuição dos respectivos incentivos (PORTUGAL, 2008).

A partir dessa publicação, passaram a ser contratualizados apenas quinze indicadores, treze comuns a todas as USF e apenas dois acordados especificamente com cada USF. Os indicadores comuns são distribuídos por quatro grandes áreas: acessibilidade (quatro), desempenho assistencial (oito), qualidade percepcionada (um) e desempenho econômico (dois). Os indicadores selecionados por cada USF pertencem ao grupo 'desempenho assistencial'. As USF com carteira adicional de serviços devem ter pelo menos um indicador de 'desempenho assistencial', que permita aferir a carga horária realizada e a produção estimada para cada atividade adicional (PORTUGAL, 2010B).

Alguns indicadores são básicos na carteira de serviços comuns a todas as USF, para atribuição dos incentivos institucionais, mesmo assim, são avaliados anualmente, a fim de verificar sua permanência ou não no processo de contratualização anual, conforme demonstrado no quadro 1. 


\begin{tabular}{|c|c|}
\hline Área & Indicadores \\
\hline Acesso & $\begin{array}{l}\text { Percentagem de consultas ao utente pelo seu próprio médico de família } \\
\text { Taxa de utilização global de consultas } \\
\text { Taxa de visitas domiciliárias médicas por } 1.000 \text { inscritos } \\
\text { Taxa de visitas domiciliárias de enfermagem por } 1.000 \text { inscritos }\end{array}$ \\
\hline Desempenho assistencial & $\begin{array}{l}\text { Percentagem de mulheres, entre } 25 \text { e } 64 \text { anos, com colpocitologia atualizada } \\
\text { Percentagem de mulheres, entre } 50 \text { e } 69 \text { anos, com mamografia registrada nos últimos dois anos } \\
\text { Percentagem de diabéticos com pelo menos duas HbA1C registradas nos últimos doze meses, desde que abranjam } \\
\text { dois semestres } \\
\text { Percentagem de hipertensos com pelo menos uma avaliação de pressão arterial em cada semestre } \\
\text { Percentagem de crianças com PNV* atualizado aos } 2 \text { anos } \\
\text { Percentagem de crianças com PNV atualizado aos } 6 \text { anos } \\
\text { Percentagem de primeiras consultas na vida efetuadas até os } 28 \text { dias } \\
\text { Percentagem de primeiras consultas de gravidez no primeiro trimestre }\end{array}$ \\
\hline Satisfação dos utentes & Percentagem de utilizadores satisfeitos/muitos satisfeitos \\
\hline Eficiência & $\begin{array}{l}\text { Custo médio de medicamentos faturados por utilizador (em PVP }{ }^{\star \star} \text { ) } \\
\text { Custo médio de } \mathrm{MCDT}^{\star \star \star} \text { faturados por utilizador }\end{array}$ \\
\hline
\end{tabular}

Fonte: BRASIL, 2012

*PNV - Programa Nacional de Vacinação

** PVP - Preço de Venda ao Público

${ }^{\star * \star}$ MCDT - Meios Complementares de Diagnóstico e Terapia

Em continuidade ao processo de reforma, foram criados, em 2008, os Agrupamentos de Centros de Saúde (ACSs), através da publicação do Decreto-lei n. ${ }^{\circ} 28$, de 22 de fevereiro de 2008b. Assim, extinguiu-se a estrutura intermediária de sub-regiões de saúde, concentrando o processo de organização e gestão dos cuidados de saúde primários nos ACEs.

A estrutura envolveu não só a organização das USF, mas a estruturação de outras unidades funcionais: Unidades de Cuidados de Saúde Personalizados (UCSP), Unidades de Cuidados na Comunidade (UCC), Unidades de Recursos Assistenciais Partilhados (Urap), Unidades de Saúde Pública (USP) e unidades ligadas diretamente ao Diretor Executivo, como a Unidade de Apoio à gestão (UAG), o gabinete do cidadão, o conselho clínico e o conselho da comunidade.

O processo de contratualização estabelecido com a USF continuou com a organização dos ACEs, porém, algumas modificações foram necessárias. A partir da criação dos ACEs, o processo de contratualização passou a ter dois momentos: uma contratualização interna entre os ACEs e as unidades que os constituem, da qual resulta a assinatura de uma Carta Compromisso; e um segundo momento, a contratualização externa entre a ARS regional e os ACEs, de que resulta um Contrato - Programa, que teve início em 2011 (PORTUGAL, 2012).

A reforma da atenção primária tem demonstrado, desde o início, resultados satisfatórios no que tange à melhoria de indicadores de saúde e satisfação da população, conforme demonstrado em pesquisas e mencionado em relatórios sobre os Cuidados Primários em Saúde da Organização Mundial de Saúde (OPSS, 2003; OMS, 2008). Porém, alguns autores destacam que a adesão à reforma não tem sido uniforme ao longo do País, criando desigualdades nos cuidados de saúde disponibilizados aos cidadãos, devido às fragilidades na distribuição e recolocação de médicos de família em algumas regiões. Prevê-se que, até 2020 , cerca de $56 \%$ dos médicos de família deixem a sua atividade profissional. Essas perdas terão implicações, como uma menor cobertura da população e a consequente sobrecarga dos níveis secundários de cuidados, assim como redução da eficiência e aumento de gastos na saúde (MIGUEL; SÁ, 2010). 
O processo que ainda decorre assenta-se numa segunda etapa de desenvolvimento e amadurecimento. A garantia da sustentabilidade dessa reforma em médio e longo prazos passa por manter as estratégias de mudança com ajustes que possam aumentar a sua implementação, autonomia e que diversifiquem a oferta de unidades, assim como a edificação de uma cultura e de práticas regulares de responsabilização, de avaliação e de transparência em todos os níveis. Consequentemente, esse é um dos desafios que se colocam para os próximos anos (PORTUGAL, 2010).

\section{Considerações para o cenário brasileiro}

Os sistemas de saúde, em geral, apontam os processos de contratualização como um instrumento necessário para alcançar uma maior eficiência dos recursos utilizados, facilidade de acessibilidade aos serviços, uma prestação de serviços de elevada qualidade, com maior enfoque na promoção da saúde e prevenção de doenças (OMS, 2006).

Porém, convém ressaltar que alguns elementos comuns de sucesso, observados em diversos países que utilizam o instrumento, compreendem a descentralização das unidades de prestação de serviços, a criação de equipes multidisciplinares, o envolvimento e a motivação dos profissionais e uma maior autonomia dos processos decisórios e da gestão de recursos para os profissionais de 'linha de frente', por se encontrarem mais próximos dos doentes, com maior conhecimento das necessidades e expectativas dos usuários. Outro fator implicado no sucesso consiste da articulação dos demais níveis de assistência à atenção primária, que potencie a integralidade do cuidado do paciente, devidamente sustentados por modelos de incentivos financeiros (ESCOVAL ET AL., 2010).

Ao analisarmos a situação do Brasil, evidencia-se a importância das lutas e dos movimentos sociais que levaram ao estabelecimento de um Sistema Único de Saúde (SUS) público; os desafios percorridos para a sua implantação, em um país de enorme dimensão territorial, com disparidades regionais e sociais; as dificuldades no processo de descentralização; o subfinanciamento do setor; a reestruturação da rede de serviços integrados e orientados para a atenção primária; as fragilidades do processo de formação; a qualificação e a inserção de recursos humanos; a ausência de regulação profissional e do trabalho; e o aumento de setores privados em concorrência com os recursos públicos.

Mesmo diante de tantos desafios, o País apresentou grande avanço nos últimos anos. A ESF, como modelo organizacional da APS, vem alcançando resultados importantes e positivos para a melhoria dos indicadores de saúde e tem possibilitado maior integração aos serviços, o redirecionamento de recursos entre as regiões do País, a implantação do Piso de Atenção Básica (PAB) e do pacto pela saúde, como proposta descentralizadora (LIMA ET AL., 2012; PAIM, 2006).

A ESF, além de expandir o acesso aos serviços da população, repercutiu na implantação de novas políticas de saúde, como: promoção de saúde, fornecimento de medicamentos, saúde bucal, implementação de estratégias de formação, qualificação e inserção profissional, políticas de avaliação e monitoramento, entre tantas outras que foram necessárias para dar os devidos suporte e 'consistência' ao modelo preconizado.

Também, ao analisarmos os caminhos percorridos sobre os processos avaliativos para a APS, sobretudo quanto às políticas de avaliação, percebe-se uma trajetória prioritária para os processos de avaliação e monitoramento voltados para a expansão da cobertura do programa, a implementação de novas equipes, de outras categorias profissionais, relacionadas aos processos de trabalho e aos produtos executados, em prol da melhoria de acesso e de indicadores de saúde. 
Já no tocante à outra vertente relacionada ao processo avaliativo, referente às políticas de avaliação de recursos humanos, de avaliação de desempenho profissional e de contratualização de metas voltadas para a melhoria da qualidade, percebe-se a existência de uma trajetória um tanto 'sombria', com poucos investimentos direcionados para a área $\mathrm{e}$ sem experiência ao longo dos últimos anos.

A proposta do PMAQ surge em 2011, em âmbito nacional, pela possibilidade de maior envolvimento dos profissionais no processo avaliativo, tanto com relação aos processos de trabalho executados quanto às políticas de recursos humanos voltadas para a atenção primária.

O PMAQ vem para impulsionar melhorias nas instalações físicas das unidades, no planejamento e na organização dos serviços, na avaliação e no monitoramento dos indicadores de saúde, em gestão, formação e qualificação profissional, além de viabilizar incentivos financeiros tanto para os municípios participantes quanto para os profissionais, em reconhecimento ao trabalho executado. Esse é um modelo que possibilita comparações tanto entre equipes do mesmo município como na esfera nacional. Para isso, requer o cumprimento de indicadores em áreas estratégicas estabelecidas, o que leva em conta um padrão de prioridade nacional.

Ressaltam-se, porém, alguns fatores que necessitam ser analisados como pontos de fragilidades que envolvem tanto a escolha dos indicadores de monitoramento selecionados pelas equipes quanto aos critérios de adesão das equipes. Quanto aos indicadores selecionados, estes podem não privilegiar as necessidades locais de saúde da população e não englobar as competências técnicas dos profissionais, podendo não ser um representativo de melhoria de qualidade. Outro aspecto refere-se ao processo de seleção e adesão das equipes, podendo ocorrer um viés pelos gestores, que passam a privilegiar as equipes com melhores condições de bons resultados para recebimento de incentivos, em detrimento de outras, para certificação, não condizendo com uma realidade global.

Em paralelo, destaca-se a ausência de uma 'cultura de avaliação' e negociação entre profissionais e gestores. Muitos profissionais sentem-se amedrontados com processos avaliativos, por não seguirem uma normatização ou pelo fato de os processos de negociação, na maioria das vezes, privilegiarem o poder da gestão política em detrimento da gestão técnica.

Outro fator que pode comprometer os processos gerenciais refere-se à alta rotatividade ainda existente, tanto de gestores como de profissionais, representando uma ameaça para a credibilidade de sistemas de avaliação, onde muitos programas são descontinuados com as mudanças de governo.

No campo do trabalho, o programa pode ser um aliado dos profissionais de saúde, possibilitando maior poder de negociação com gestores e discussões sobre salários, vínculos trabalhistas e plano de carreiras, e favorecedor de fixação profissional e carreira para o setor.

O estudo de caso realizado em Portugal possibilitou a identificação de aspectos positivos que poderão servir de reflexão ao cenário brasileiro:

A importância de uma gestão profissional próxima aos serviços e à população, possibilitando adequações, com rapidez, dos serviços às necessidades das populações;

A importância condicionada à gestão e à governação clínica na APS, com formação específica dos diretores executivos. Mais ainda, a discussão de normas de orientação clínica e a participação da comunidade nos conselhos;

A importância da integração entre a avaliação de desempenho profissional e a contratualização como um caminho a percorrer em prol de melhorias no sistema;

A trajetória de implantação de um modelo, que já ocorre há alguns anos, até o aperfeiçoamento dos instrumentos, a adequação do sistema e a imersão em uma 'cultura'; 
A capacitação do processo de contratualização e apoio aos serviços e o monitoramento rigoroso das ARS aos ACEs.

A análise do modelo de APS de Portugal revelou pontos importantes, que servem de análise para o programa instituído na ESF no Brasil. A APS, no Brasil, avançou muito positivamente em vários aspectos, entretanto, claramente, será necessário maior empenho do governo federal, dos estados e municípios em:

- Avançar na discussão sobre o processo de descentralização/regionalização;

- Garantir maior autonomia aos gestores e unidades de saúde da família;

- Rediscutir o financiamento da atenção primária e o incremento e a autonomia de utilização de recursos financeiros em âmbito local;

- Avançar nas discussões sobre os recursos humanos em saúde, com estratégias para formação e qualificação, estimular a atração e a fixação de profissionais - já que a APS ainda apresenta alta rotatividade de profissionais;

- Investir na formação de agentes comunitários de saúde, que, em muitas ocasiões, são subaproveitados. Em certas localidades, poderiam trazer ganhos à saúde da população, se bem treinados;

- Implementação da governança local e fortalecimento dos conselhos gestores.

\section{Conclusão}

Este artigo possibilitou discutir alguns aspectos importantes sobre sistemas de avaliação e contratualização profissional no âmbito da atenção primária, com suas trajetórias, tendências, fragilidades, limitações, avanços alcançados. Contudo, é de se mencionar a necessidade de investimentos e estudos nessa área diante da extrema importância que os processos avaliativos propiciam para a melhoria da qualidade dos sistemas de saúde.

A análise de uma experiência aponta que enfrentar mudanças é necessário, diante de um processo de globalização, de oscilações dos planos econômico e político e das transições demográfica e epidemiológica por que, constantemente, passamos ao longo dos anos. Avaliar as iniciativas tomadas sobre as reformas pode auxiliar a enfrentar as transformações, sempre em busca da melhor resposta para o que a população necessita. 


\section{Referências}

BRANCO, A. G.; RAMOS, V. Cuidados de saúde primários em Portugal. Revista Portuguesa de Saúde Pública, Lisboa, v. 2, p. 5-12, 2001.

BRASIL. Ministério da Saúde. Secretaria de Atenção à Saúde. Departamento de Atenção Básica. Atenção Básica e a Saúde da Família. Disponível em: <http:// www.saude.gov.br/dab>. Acesso em: 01 out. 2011a.

Ministério da Saúde. Secretaria de Atenção à Saúde. Departamento de Atenção Básica. Portaria n. ${ }^{\circ} 1654$, de 19 de julho de 2011. Institui, no âmbito do Sistema Único de Saúde, o Programa Nacional de Melhoria do Acesso e da Qualidade da Atenção Básica (PMAQ-AB) e o Incentivo Financeiro do PMAQ-AB. Brasília. Diário Oficial [da] República Federativa do Brasil, Brasília, DF, 2011b.

Ministério da Saúde. Secretaria de Atenção à Saúde. Departamento de Atenção Básica. Teto, credenciamento e implantação das estratégias de Agentes Comunitários de Saúde, Saúde da Família e Saúde Bucal Unidade Geográfica: Brasil Competência: Junho de 2012 a Julho de 2012. Disponível em: <http://dab.saude.gov.br/ historico_cobertura_sf.php>. Acesso em: 09 ago. 2012.

CASTRO, A. L. B.; MACHADO, C. V. A política federal de atenção básica à saúde no Brasil nos anos 2000. Physis, Rio de Janeiro, v. 22, n. 2, p. 477-506, jun. 2012.

ESCOVAL, A.; RIBEIRO, R. S.; MATOS, T. T. A contratualização em cuidados de saúde primários: o contexto internacional. Revista Portuguesa de Saúde Pública, Lisboa, v. 9, p. 41-57, 2010.

GIRARDI, S. N. et al. Pesquisa nacional de Monitoramento da Qualidade do Emprego na Estratégia Saúde da Família. Relatório de Pesquisa - Universidade Federal de Minas Gerais, Faculdade de Medicina, Núcleo de Educação em Saúde Coletiva, Estação de Pesquisa de Sinais de Mercado. Belo Horizonte, 2009.

KALINICHENKO, O. et al. Performance assessment in Primary Health Care: a System Literature Review. Portugal. University of Algarve- faculty of Economics and CEFAGE-UE. 2013, 17 p.

LAPÃO, L. V.; DUSSAULT, G. From policy to reality: clinical managers' views of the organizational challenges of primary care reform in Portugal.

The International journal of health planning and management, v. 27, n. 4, p. 295-307, 2012.

LIMA, L. et al. Descentralização e regionalização: dinâmica e condicionantes da implantação do Pacto pela Saúde no Brasil. Ciência \&t Saúde Coletiva, Rio de Janeiro, v. 17, n. 7, p. 1903-1914, 2012.

MACINKO, J. et al. Going to scale with community-based primary care: an analysis of the family health program and infant mortality in Brazil, 1999-2004. Social science \&t medicine, London, v. 65, n. 10, p. 20702080, nov. 2007

MENDONÇA, C. S. Uso das Internações por condições sensíveis à Atenção Primária: O caso de Belo Horizonte/MG. Dissertação (Mestrado Profissional em Epidemiologia) - Universidade Federal do Rio Grande do Sul, Porto Alegre, 2009.

MIGUEL, L. S.; SÁ, A. B. Cuidados de Saúde Primários em 2011-2016: reforçar, expandir-Contribuição para o Plano Nacional de Saúde 2011-2016. Lisboa: Ministério, 2010.

NEY, M. S; RODRIGUES, P. H. A. Fatores críticos para fixação do médico na Estratégia Saúde da Família. Physis: Revista de Saúde Coletiva, Rio de Janeiro, v. 22, n. 4, p.1293-1311, 2012.

\section{OBSERVATÓRIO PORTUGUÊS DE SISTEMAS} DE SAÚDE (OPSS). Evolução do Sistema de saúde Português (internet). Rede de investigadores do OPSS. p. 4, 2003. Disponível em: <www.observaport.org>. Acesso em: 01 fev. 2013.

ORGANIZAÇÃO MUNDIAL DA SAÚDE (OMS). Relatório Mundial da Saúde 2006: Trabalhando juntos pela saúde, 2006 .

PAIM, J. S. Equidade e reforma em sistemas de serviços de saúde: o caso do SUS. Saúde e Sociedade, São Paulo, v. 15, n. 2, p. 34-46, 2006.

PISCO, L. A reforma dos cuidados de saúde primários Cadernos de Economia, Chapecó, v. 20, n. 80, p. 60-66, 2007.

Reforma da Atenção Primária em Portugal em duplo movimento: unidades assistenciais autônomas de saúde familiar e gestão em agrupamentos de Centros de Saúde. Ciência \&t Saúde Coletiva, Rio de Janeiro, v. 16, n. 6, p. 2841-52, 2011. 
PORTUGAL. Governo da República Portugues. Revisão histórica do Serviço Nacional de Saúde de Portugal [internet]. 2012a. Disponível em: <http://www.portugal.gov.pt/pt/os-ministerios/ministerio-da-saude/ quero-saber-mais/quero-aprender/historia-sns.aspx>. Acesso em: 16 nov. 2012.

. Ministério das Finanças e da Administração Pública e da Saúde. Portaria n. ${ }^{\circ} 301 / 2008$, de 18 de Abril. Regula os critérios e condições para atribuição de incentivos institucionais e financeiros às Unidades de Saúde Familiar e aos profissionais que as integram, com fundamento em melhorias de produtividade, efectividade e qualidade dos cuidados prestados. Diário da República, Lisboa, $1^{\text {a }}$ Série, n.77, 2008.

Ministério da Saúde. Alto Comissariado

de Saúde. Plano nacional de Saúde 2011-2016.

Levantamento de determinações e recomendações da Organização Mundial de Saúde, 2010a. Disponível em: <www.acs.min-saude.pt>. Acesso em: 25 nov. 2012.

Ministério da Saúde. Departamento de

Contratualização das Administrações Regionais de Saúde (DCARS). Cuidados de Saúde primários. Metodologia de Contratualização. p. 38, dez., 2010b. Disponível em: <www.acss.min-saude.pt>. Acesso em: 05 jun. 11.

Ministério da Saúde. Departamento de Contratualização das Administrações Regionais de Saúde (DCARS). Cuidados de Saúde primários. Metodologia de Contratualização. p. 20, mar.2012. Disponível em: $<$ http://www.acss.min-saude.pt/Portals/0/14_Mar_2012_ MetodologiaContratualizaoCSP_2012.pdf>. Acesso em: 20 jan. 2015.

Ministério da Saúde. Decreto-lei no 117/98, de 5 de Maio, Regime Remuneratório Experimental dos Médicos da Carreira de Clínica Geral. Diário da República, 1. a serie A, No 103, de 05.05., p. 1991, 1998. Disponível em: <http://dre.tretas.org/dre/92514/>. Acesso em 21 jan. 2015.

Ministério da Saúde. Cuidados de Saúde Primários. Guião de apoio à preparação de candidaturas a Unidades de Saúde Familiar (USF) - Modelo A., p. 19, 2010.
Ministério da Saúde. Minuta de Contrato-

Programa ARS-ACES. Disponível em: <http://www. acss.min-saude.pt/Portals/0/Minuta_CP_ACES_2010. pdf >. Acesso em: 01 abr. 2012.

Ministério das Finanças e da Administração Pública e da Saúde. Decreto - Lei n. ${ }^{\circ}$ 28/ 2008, de 28 de fevereiro de 2008. Diário da República, 28 fev, 2008b. Disponível em: <https://dre.pt/application/dir/ pdf1sdip/2008/02/03800/0118201189.PDF > . Acesso em: 01 abr. 2012

Resolução do Conselho de Ministros n. ${ }^{\circ}$ $157 / 2005$, de 12 de outubro de 2005. Institui a criação de uma estrutura de missão para a reforma dos cuidados de saúde primários. Diário da República, v.157, 2005. Disponível em: <www.mcsp.min-saude.pt/>. Acesso em: 01 abr. 2012.

SOUSA, P. A. F. O sistema de saúde em Portugal: realizações e desafios. Acta Paulista de Enfermagem, Lisboa, v. 22, n. especial, p. 884-94, 2009.

VAN LERBERGHE, W. The world health report 2008: primary health care: now more than ever. World Health Organization, 2008.

VIANA, A.L, DAL POZ, M. R. A reforma do sistema de saúde no Brasil e o Programa Saúde da Família. Physis - Revista de Saúde Coletiva, Rio de Janeiro, v. 8, n. 2, p. 11-48, 1998.

VICTORA, C. G et al. Condições de saúde e inovações nas políticas de saúde no Brasil: o caminho a percorrer. Lancet, Londres, supl. Saúde no Brasil, v. 377, n. 9782, p. 90-102, 2011.

Recebido para publicação em abril de 2014

Versão final em outubro de 2014

Conflito de interesse: inexistente

Suporte financeiro: não houve 